\title{
Formation of Hydrogen Impurity States in Silicon and Insulators at Low Implantation Energies
}

\author{
T. Prokscha, ${ }^{1, *}$ E. Morenzoni, ${ }^{1}$ D. G. Eshchenko, ${ }^{2,1}$ N. Garifianov,${ }^{3,1}$ H. Glückler, ${ }^{1, \dagger}$ R. Khasanov, ${ }^{1,2}$ \\ H. Luetkens, ${ }^{1}$ and A. Suter ${ }^{1}$ \\ ${ }^{1}$ Paul Scherrer Institut, Labor für Myon-Spin Spektroskopie, CH-5232 Villigen PSI, Switzerland \\ ${ }^{2}$ Physik Institut der Universität Zürich, CH-8057 Zürich, Switzerland \\ ${ }^{3}$ Kazan Physicotechnical Institute, RAS, Kazan 420029, Russia
}

(Received 13 July 2006; published 29 May 2007)

\begin{abstract}
The formation of hydrogenlike muonium $(\mathrm{Mu})$ has been studied as a function of implantation energy in intrinsic Si, thin films of condensed van der Waals gases $\left(\mathrm{N}_{2}, \mathrm{Ne}, \mathrm{Ar}, \mathrm{Xe}\right)$, fused and crystalline quartz, and sapphire. By varying the initial energy of positive muons $\left(\mu^{+}\right)$between 1 and $30 \mathrm{keV}$ the number of electron-hole pairs generated in the ionization track of the $\mu^{+}$can be tuned between a few and several thousand. The results show the strong suppression of the formation of those Mu states that depend on the availability of excess electrons. This indicates that the role of $\mathrm{H}$-impurity states in determining electric properties of semiconductors and insulators depends on the way in which atomic $\mathrm{H}$ is introduced into the material.
\end{abstract}

DOI: 10.1103/PhysRevLett.98.227401

PACS numbers: 78.70. $-\mathrm{g}, 36.10 . \mathrm{Dr}, 76.75 .+\mathrm{i}$

The implantation of energetic (mega-electron-volt) positive muons $\left(\mu^{+}\right)$in insulators or semiconductors commonly leads to the formation of the hydrogenlike bound state muonium $\left[\mathrm{Mu}=\left(\mu^{+} e^{-}\right)\right]$with a final charge state which can be either positive $\left(\mathrm{Mu}^{+}\right)$, neutral $\left(\mathrm{Mu}^{0}\right)$, or negative $\left(\mathrm{Mu}^{-}\right)$. In semiconductors $\mathrm{Mu}$ is used to identify and investigate the electronic properties and the behavior of isolated hydrogenlike states [1-3], and hydrogenrelated impurities which are of fundamental and technological interest due to their influence on the electrical and optical properties. Isolated $\mathrm{H}$ atoms in materials are difficult to detect by other spectroscopic means, which mostly require high $\mathrm{H}$ concentrations (see [4,5] and references therein for examples of vibrational spectroscopy studies of $\mathrm{Si}$ ). In contrast, $\mathrm{Mu}$ - which behaves like a light $\mathrm{H}$ isotope $\left(m_{\mu} \simeq m_{p} / 9\right)$ - can be easily detected and characterized by the muon spin rotation $(\mu \mathrm{SR})$ technique due to its high sensitivity per spin. Muonium states, formed after implantation of energetic $\mu^{+}$, remain isolated during the observational time window of the order of the $\mu^{+}$lifetime $(2.2 \mu \mathrm{s})$. Therefore, a large amount of experimental information on the formation, structure and electrical activity of isolated $\mathrm{H}$ states in semiconductors has been obtained from $\mu \mathrm{SR}$, which has played a pioneering role in the identification and characterization of hydrogenlike states. In $\mathrm{Si}, \mathrm{Ge}$, and semiconductors of the III-V family two Mu states lying deep in the band gap have been identified at low temperatures $(<50 \mathrm{~K})$ [1]: normal $\mathrm{Mu}_{T}$ in the tetrahedral interstitial site with a large isotropic hyperfine interaction (HFI) and anomalous $\mathrm{Mu}_{\mathrm{BC}}$ at a bond center between two host atoms with a smaller, anisotropic HFI. In covalent semiconductors, $\mathrm{Mu}_{T}$ acts as an acceptor and $\mathrm{Mu}_{\mathrm{BC}}$ as a donor. Recently, novel, very weakly bound $\mathrm{Mu}$ states (shallow $\mathrm{Mu}$, binding energies $15-60 \mathrm{meV}$ ) with very low HFI have been established in a number of II-VI and III-V (nitrides) compounds [6-8]. Theoretical work has shown a universal alignment of hydrogen levels in semiconductors and insulators [9], from which the electronic properties of hydrogen impurities can be derived. The predicted shallow donor hydrogen states in $\mathrm{InN}$ and $\mathrm{ZnO}$ have been confirmed experimentally by $\mu$ SR $[7,8]$.

However, it has to be kept in mind that by the techniques used so far the spectroscopically investigated $\mathrm{H}$ isotopes are energetically inserted in the solid. This results in a large number $N_{e h}$ of electron-hole pairs generated during slowing down of the incident particle. For instance, all $\mu \mathrm{SR}$ experiments performed up to now used $\mathrm{MeV}-\mu^{+}$beams that generate $10^{5}-10^{6}$ electron-hole pairs per implanted $\mu^{+}$in the ionization track [10]. Similar or higher numbers of excess $e^{-}$are created by the implantation of $\mathrm{H}$ or $\mathrm{D}$ ions used in the case of vibrational spectroscopy or channeling experiments. A sizable fraction of these electron-hole pairs escapes prompt recombination and is still present around the thermalized impurity as shown in $\mu$ SR experiments with applied electric field $\mathbf{E}$ of both polarities. The $\mathbf{E}$ field clearly changes the $\mathrm{Mu}$ formation probability by pushing track $e^{-}$and $\mu^{+}$apart or together [11-14], demonstrating that a significant fraction of $\mathrm{Mu}$ in semiconductors and insulators is generated by the capture of a track $e^{-}$after the $\mu^{+}$has stopped at an interstitial or bond site. In semiconductors it appears that this so-called delayed $\mathrm{Mu}$ formation (in contrast to prompt $\mathrm{Mu}$, where $\mathrm{Mu}$ forms during slowing down in charge-exchange cycles, followed by thermalization of Mu due to elastic collisions [14]) is the origin of $\mathrm{Mu}_{\mathrm{BC}}$ and for the recently discovered shallow $\mathrm{Mu}$ centers in III-V and II-VI semiconductors [12,13,15]. The question therefore arises whether and how the final states are influenced by the formation process, which is essential for studies on technologically important semiconductors and insulators. This can be studied by using the polarized low-energy $\mu^{+}\left(\mathrm{LE}-\mu^{+}\right)$beam at the Paul Scherrer Institute (PSI, Villigen, Switzerland) $[16,17]$ with variable 
implantation energy between 1 and $30 \mathrm{keV}$. It allows one to investigate the formation of hydrogenlike $\mathrm{Mu}$ impurity states as a function of energy, i.e., as a function of $N_{e h}$. By varying the energy, $N_{e h}$ can be tuned between a few and several thousand. This is up to 5 orders of magnitude less than for conventional MeV-muon beams. Below $1 \mathrm{keV}$ nearly no track products are generated, thus approximating the case where $\mathrm{H}$ impurities are thermally introduced, which is how trace atoms are incorporated in the lattice in the course of wafer growth and fabrication processes.

In this Letter we investigate for the first time the formation of thermal $\mathrm{Mu}$ as a prototype for isolated $\mathrm{H}$ impurities as a function of implantation energy. In addition to intrinsic $\mathrm{Si}$ and sapphire $\left(\mathrm{Al}_{2} \mathrm{O}_{3}\right)$ with more than one type of $\mathrm{Mu}$ we investigated thin films of van der Waals solids $\left(s\right.$-Ne, $s$-Ar, $\left.s-\mathrm{Xe}, s-\mathrm{N}_{2}\right)$ and fused and crystalline quartz $\left(\mathrm{SiO}_{2}\right)$ due to their simplicity concerning the final charge states: only one type of $\mathrm{Mu}^{0}$ exists with an isotropic HFI close to vacuum $\mathrm{Mu}^{0}$. We find that delayed $\mathrm{Mu}$ formation is energy dependent in the $\mathrm{keV}$ range in all the investigated samples. Below $\sim 10 \mathrm{keV}$ the formation of those $\mathrm{H}$ impurity states that require a sizable amount of excess $e^{-}$is strongly suppressed. The data on $\mathrm{Si}$ and $\mathrm{Al}_{2} \mathrm{O}_{3}$ support the interpretations that $\mathrm{Mu}_{\mathrm{BC}}^{0}$ in $\mathrm{Si}$ [12] and $\mathrm{Mu}^{-}$ in $\mathrm{Al}_{2} \mathrm{O}_{3}$ [18] are formed by delayed capture of a track $e^{-}$.

The $\mu$ SR technique allows one to differentiate between paramagnetic $\left(\mathrm{Mu}^{0}\right)$ and $\mu^{+}$in diamagnetic environment (free $\mu^{+}, \mathrm{Mu}^{+}$, or $\mathrm{Mu}^{-}$). Because of the hyperfine coupling between the $\mu^{+}$and the $e^{-}$spin the observable Larmor precession frequency of isotropic $\mathrm{Mu}^{0}$ is about 103 times larger than for the free $\mu^{+}$. It splits into two intratriplet lines that merge to one line at low fields $(<2 \mathrm{mT})$ where $50 \%$ of the muon polarization is not observed due to unresolved hyperfine oscillations between the triplet and singlet state. The diamagnetic and paramagnetic decay asymmetries $A_{D}$ and $A_{\mathrm{Mu}}$ were determined by measuring the amplitudes of the $\mu^{+}$and $\mathrm{Mu}$ precession signals in transverse (perpendicular to the $\mu^{+}$spin) magnetic field, applied parallel to the sample normal. $A_{D}$ and $A_{\mathrm{Mu}}$ are proportional to the fraction of muons in that particular state.

The $0.5 \mathrm{~mm}$ thick Si sample with $50 \mathrm{~mm}$ diameter (undoped, resistivity $10 \mathrm{k} \Omega \mathrm{cm}$, capped by a $2 \mathrm{~nm}$ thick oxide layer) was oriented with the $\langle 100\rangle$ direction parallel to the sample normal. The quartz disc samples had thicknesses of 1 and $2 \mathrm{~mm}\left[\mathrm{SiO}_{2}\right.$ crystal and fused quartz (Suprasil), respectively] and $50 \mathrm{~mm}$ diameter. The $\mathrm{Al}_{2} \mathrm{O}_{3}$ sample was a $0.5 \mathrm{~mm}$ thick single crystal with $60 \mathrm{~mm}$ diameter. The solid gas films were grown at partial pressures between $10^{-6}$ and $5 \times 10^{-5} \mathrm{hPa}$. Film thicknesses were about $1000 \mathrm{~nm}$ which is sufficient to stop all LE- $\mu^{+}$ in the layer. At these deposition pressures grain sizes of order $100 \mathrm{~nm}$ are obtained [19]. For details on the experimental setup we refer to Ref. [20].

Figure 1 shows typical $\mu \mathrm{SR}$ asymmetry spectra, and displays the results for $\mathrm{Si}$. In $\mathrm{Si}$, at $5 \mathrm{mT}$ only the precession
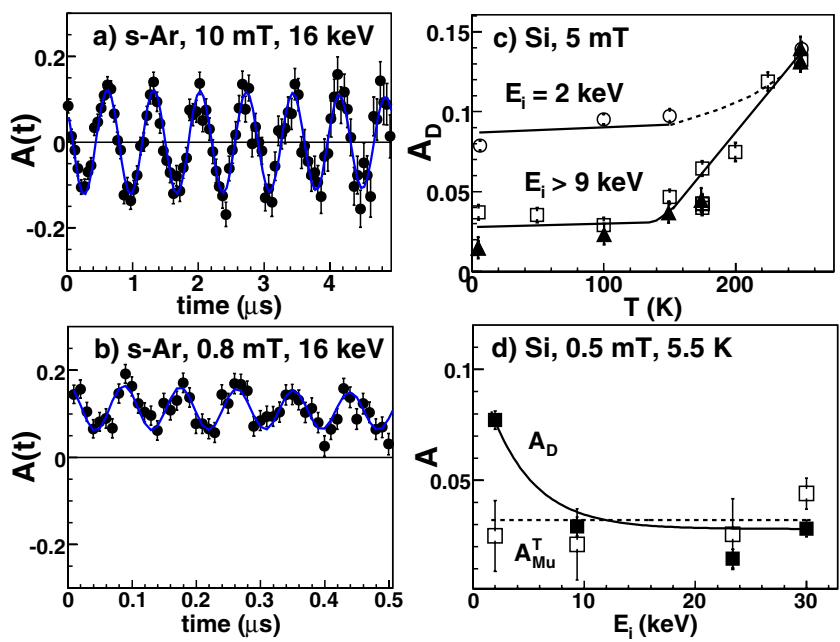

FIG. 1 (color online). (a) Typical $\mu \mathrm{SR}$ asymmetry spectrum $A(t)$ for the diamagnetic signal in $s$-Ar, and (b) corresponding signal at low fields showing the 103 times faster $\mathrm{Mu}$ precession superposed to the slow diamagnetic signal. (c) Undoped $\mathrm{Si}$, diamagnetic asymmetry $A_{D}$ as a function of temperature $T$. Solid triangles: implantation energy $E_{i}>20 \mathrm{keV}$; open squares: $E_{i}=9.3 \mathrm{keV}$; open circles: $E_{i}=2.0 \mathrm{keV}$. (d) $A_{D}$ and $\mathrm{Mu}_{T}$ asymmetry $A_{\mathrm{Mu}}^{T}$ as a function of $E_{i}$. The lines in (a) and (b) are fits, and in (c) and (d) are guides to the eye.

of the diamagnetic signal is observed. Because of limited statistics and time resolution $\mathrm{Mu}$ precession frequencies $>30 \mathrm{MHz}$ are too high to be resolved with our present setup. In a field of $5 \mathrm{mT}$, the $\mathrm{Mu}_{T}^{0}$ intratriplet lines are at about $70 \mathrm{MHz}$, whereas the $\mathrm{Mu}_{\mathrm{BC}}^{0}$ transitions are between 35 and $50 \mathrm{MHz}$, depending on the orientation of the $\mathbf{B}$ field with respect to the $\langle 111\rangle$ crystal axis. At $0.5 \mathrm{mT}$ the $\mathrm{Mu}_{\mathrm{BC}}^{0}$ frequencies are nearly unchanged and therefore not observable with our setup, whereas the $7 \mathrm{MHz}$ signal of $\mathrm{Mu}_{T}^{0}$ becomes visible. The $0.5 \mathrm{mT}$ data are fitted with two components, a $\mu^{+}$precession signal and the $\mathrm{Mu}_{T}^{0}$ signal with exponential relaxation, whereas the $5 \mathrm{mT}$ data are fitted with the $\mu^{+}$precession signal only. The temperature dependence of $A_{D}$ at different implantation energies $E_{i}$ is shown in Fig. 1(a). Above $9 \mathrm{keV}$-corresponding to a mean implantation depth $\langle d\rangle$ of $65 \mathrm{~nm}$ and $N_{e h} \simeq 2400$ [10] $-A_{D}$ exhibits the same temperature behavior as in bulk $\mu$ SR experiments [12]. The increase of $A_{D}$ above $\sim 150 \mathrm{~K}$ reflects the thermally induced ionization of $\mathrm{Mu}_{\mathrm{BC}}^{0}$. At $E_{i}=2 \mathrm{keV}(\langle d\rangle \sim 18 \mathrm{~nm})$ and $T<150 \mathrm{~K} A_{D}$ is significantly larger than at higher energies. As Fig. 1(b) shows the behavior of $A_{D}$ is not related to a change of the $\mathrm{Mu}_{T}^{0}$ fraction, which, contrary to $A_{D}$, does not depend on $E_{i}$. It rather reflects the unobserved $\mathrm{Mu}_{\mathrm{BC}}^{0}$ fraction which decreases with decreasing energy and number of available track $e^{-}$. This is also supported by the convergence of the two curves in Fig. 1(a) at high $T$ where $\mathrm{Mu}_{\mathrm{BC}}^{0}$ is ionized. The $\sim 2 \mathrm{~nm}$ thick oxide layer present on the Si surface is too thin to explain the observed reduction of the $\mathrm{Mu}$ formation. Moreover, in $\mathrm{SiO}_{2}$ layer at low energy a higher 
Mu fraction should be observed; see Fig. 2(b). The different dependence on the availability of excess $e^{-}$indicate that the main fraction of $\mathrm{Mu}_{\mathrm{BC}}^{0}$ is due to delayed formation, whereas $\mathrm{Mu}_{T}^{0}$ is a consequence of charge-exchange processes at epithermal energies - in agreement with bulk $\mu$ SR studies, where an applied $\mathbf{E}$ field was used to vary the average distance between $\mu^{+}$and excess $e^{-}$, and therefore the relative formation probability of these two states [12].

Figure 2 shows the energy dependence of $A_{D}$ and $A_{\mathrm{Mu}}$ for $s-\mathrm{Ar}[2(\mathrm{a})]$ and $\mathrm{SiO}_{2}$ [2(b)]. Only isotropic $\mathrm{Mu}$ is present, and $A_{D}$ and $A_{\mathrm{Mu}}$ represent a direct measure of the $\mu^{+}$and $\mathrm{Mu}$ fraction in the sample. The sum $A_{\text {tot }}=$ $A_{D}+2 A_{\mathrm{Mu}}=0.263(1)$ is the total observable asymmetry, and there is within the experimental errors no missing fraction. Qualitatively, the $s$ - $\mathrm{Ar}$ and $\mathrm{SiO}_{2}$ data display the same behavior: with increasing energy $A_{D}$ is decreasing while $A_{\mathrm{Mu}}$ is increasing correspondingly. The energydependent diamagnetic fractions $F_{D}=A_{D} / A_{\text {tot }}$ for various insulators are summarized in Fig. 3. With the exception of $s$-Ne all samples show a decreasing diamagnetic fraction with increasing energy. For $\mathrm{SiO}_{2}$ and $s$-Xe bulk Mu fractions $F_{\mathrm{Mu}}=\left(1-F_{D}\right)$ of $85 \%$ and $\sim 100 \%$, respectively, are obtained at $20 \mathrm{keV}$ [corresponding to $\langle d\rangle=155 \mathrm{~nm}$ $\left.\left(\mathrm{SiO}_{2}\right),\langle d\rangle=185 \mathrm{~nm}(s-\mathrm{Xe})\right]$. At this energy the number of electron-hole pairs created in the ionization track is

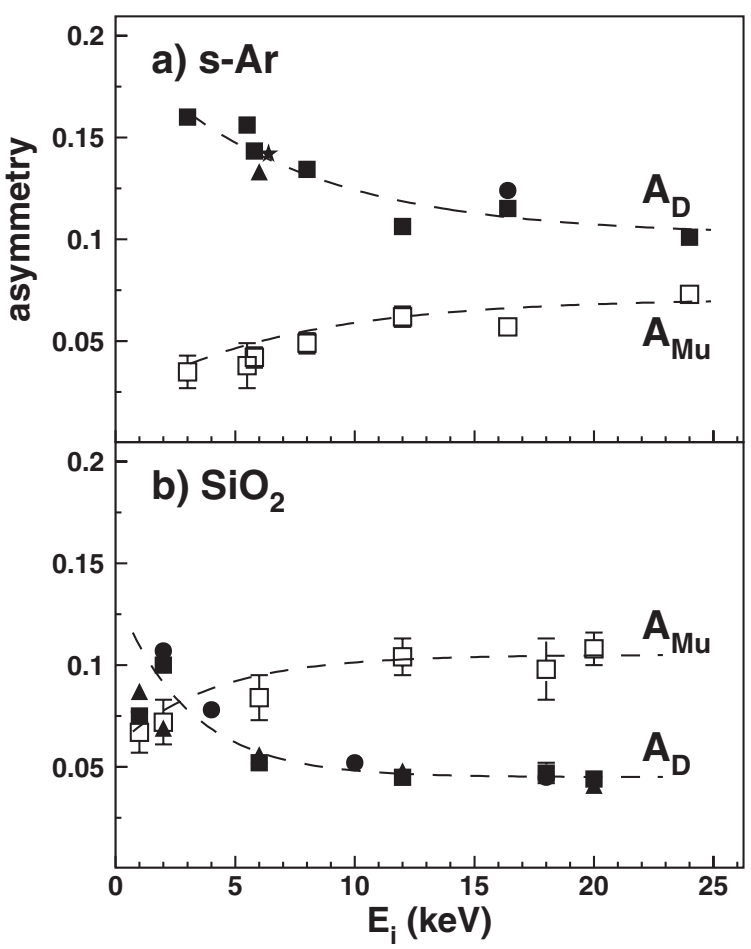

FIG. 2. Muon and Mu asymmetries $A_{D}$ and $A_{\mathrm{Mu}}$ as a function of implantation energy $E_{i}$ for (a) $s$-Ar grown at $6.5 \times 10^{-6} \mathrm{hPa}$ and (b) $\mathrm{SiO}_{2}$ crystal and glass (Suprasil), $T=20 \mathrm{~K}$. The magnetic fields are $10 \mathrm{mT}$ (circles), $5 \mathrm{mT}$ (triangles), $2 \mathrm{mT}$ (stars), and $0.8 \mathrm{mT}$ (squares). The lines are guides to the eye. about 1000 [10]. In the $s$-Ar and $s-\mathrm{N}_{2}$ films even at the highest energy the observed Mu fractions $\left(F_{\mathrm{Mu}} \sim 60 \%\right)$ are lower than the bulk results obtained with $4-\mathrm{MeV} \mu^{+}$ $\left[F_{\mathrm{Mu}} \sim 100 \%(s-\mathrm{Ar}), F_{\mathrm{Mu}} \sim 80 \%\left(s-\mathrm{N}_{2}\right.\right.$ at $\left.\left.T<30 \mathrm{~K}\right)\right]$. The discrepancy is even more drastic for $s$-Ne where the film data are consistent with $F_{\mathrm{Mu}}=0$ in contrast to the bulk data with $F_{\mathrm{Mu}}=90 \%$ [14]. This disagreement can be explained by the suppression of Mu formation in granular $s$-Ne, $s$-Ar and $s-\mathrm{N}_{2}$ thin films, as we discuss below.

The decrease of $F_{D}$ with increasing $E_{i}$ reflects the onset of delayed $\mathrm{Mu}$ formation with increasing availability of excess $e^{-}$. From the flattening of $F_{D}$ at $\sim 20 \mathrm{keV}$ we estimate the number of excess $e^{-}$necessary to saturate the delayed Mu yield to be of the order of thousand. The $e^{-}$ may escape recombination with the $\mu^{+}$by several processes: recombination with a cation from the ion track, trapping at grain boundaries, voids, and surfaces or escape from the surface ( $e^{-}$escape depth $\sim 100 \mathrm{~nm}$ in $s$-Ar and $s$-Xe [21], $e^{-}$mean free path in $\mathrm{Si}$ is $\sim 20 \mathrm{~nm}$ at $300 \mathrm{~K}$, increasing to $>100 \mathrm{~nm}$ at lower $T$ ). An additional obstacle for electron-muon recombination is also the large escape depth of 20-100 nm of epithermal $\mu^{+}$in wide band gap insulators such as $s-\mathrm{N}_{2}, s-\mathrm{Ar}$, and $s$-Ne [22]: after leaving the charge-exchange cycles where the last $e^{-}$are released the $\mu^{+}$may move such a distance away from its ionization track, further losing energy inefficiently by elastic collisions. This large $e^{-}-\mu^{+}$separation and the trapping of $e^{-}$combine all together to suppress the delayed $\mathrm{Mu}$ formation channel in $s-\mathrm{Ne}, s-\mathrm{Ar}$, and $s-\mathrm{N}_{2}$. The total suppression of Mu formation in $s-\mathrm{Ne}$ is probably a consequence of a $\mu^{+}$escape depth larger than the typical grain size, making the formation of a delayed $e^{-}-\mu^{+}$bound state unlikely.

The energy dependence at $T<100 \mathrm{~K}$ of $A_{D}$ in sapphire (Fig. 4) shows an interesting anomaly compared to the data

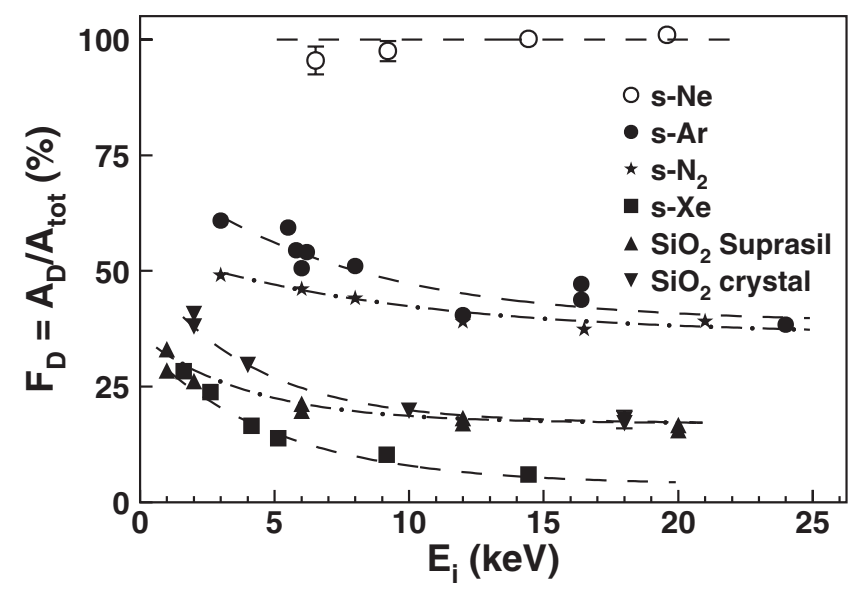

FIG. 3. Comparison of the diamagnetic fraction $F_{D}$ as a function of implantation energy $E_{i}$ for different samples, $B=$ $10 \mathrm{mT}$. Deposition pressures were $7 \times 10^{-6} \mathrm{hPa}$ for $s$-Ne, $6.5 \times$ $10^{-6} \mathrm{hPa}$ for $s$-Ar, $2.2 \times 10^{-5} \mathrm{hPa}$ for $s-\mathrm{N}_{2}$, and $1.5 \times$ $10^{-5} \mathrm{hPa}$ for $s$-Xe. The lines are guides to the eye. 


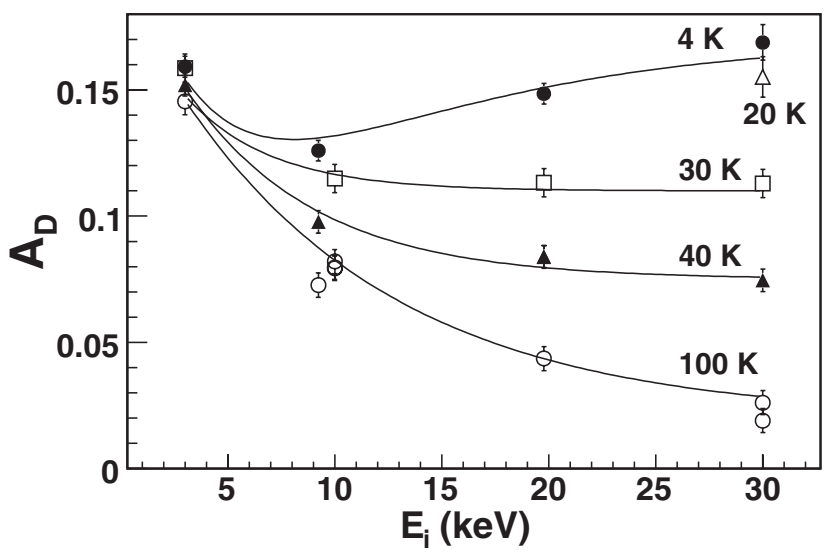

FIG. 4. Diamagnetic asymmetry $A_{D}$ as a function of implantation energy $E_{i}$ for sapphire, measured at $B=10 \mathrm{mT}$ at different temperatures. The lines are guides to the eye.

presented so far. At $100 \mathrm{~K} A_{D}$ decreases with increasing energy and reaches its smallest value of 0.025 at $30 \mathrm{keV}$. This behavior correlates with the onset of delayed formation of $\mathrm{Mu}^{0}$ as seen in other insulators. The energy dependence of $A_{D}$ becomes less pronounced on reducing the temperature. At $4 \mathrm{~K} A_{D}$ exhibits a minimum at $10 \mathrm{keV}$ and starts to increase again when further increasing the energy. This may reflect the delayed formation of diamagnetic $\mathrm{Mu}^{-}$, as suggested in a previous $\mathbf{E}$-field $\mu$ SR experiment where the disappearance of $\mathrm{Mu}^{-}$with increasing $T$ is interpreted as thermal ionization of $\mathrm{Mu}^{-}$with an activation temperature of $130 \mathrm{~K}$ [18]. A recent theoretical work shows that $\mathrm{H}^{-}$could be the stable charge state in $\mathrm{Al}_{2} \mathrm{O}_{3}$ [23]. Our data support this idea, and that $\mathrm{Mu}^{-}$is formed by delayed $e^{-}$capture.

In conclusion, the measured energy dependence of $\mathrm{Mu}$ formation in intrinsic $\mathrm{Si}$ and insulators shows as a general behavior that the formation of delayed $\mathrm{Mu}$ states requires the presence of the order of thousand excess $e^{-}$in the ionization track. With LE- $\mu^{+} \mathrm{H}$-impurity states can be studied without the generation of a nonequilibrium electron track. From the implantation energies involved we infer that the length scale of that part of the track that is involved in delayed $\mathrm{Mu}$ formation is of the order of $100 \mathrm{~nm}$. At energies $<3 \mathrm{keV}$ delayed $\mathrm{Mu}$ formation is nearly absent. This indicates that the formation of those $\mathrm{H}$-impurity states which heavily depend on the availability of excess $e^{-}$is strongly suppressed in cases where the $\mathrm{H}$ isotope is inserted in the solid without the concomitant presence of a sizable number of excess $e^{-}$. This implies, that the role of $\mathrm{H}$-impurity states in determining electric properties of semiconductors and insulators depends on how atomic $\mathrm{H}$ is incorporated into the material. The question of the relative importance of different possible $\mathrm{H}$ states and their occurrence as native impurity states in semiconductors and insulators is generally not addressed and we hope that our results will foster new theoretical and experimental studies in this area.
We are extending this kind of experiment to the investigation of shallow $\mathrm{Mu}$ states: Preliminary data in $\mathrm{ZnO}$ show also a decreasing shallow $\mathrm{Mu}$ fraction with decreasing implantation energy, which further supports the general trend found in other materials.

This work was fully performed at the Swiss Muon Source $\mathrm{S} \mu \mathrm{S}$, Paul Scherrer Institute, Villigen, Switzerland. We are grateful to S. F. J. Cox for valuable discussions. We thank C. David from LMN at PSI for providing the $\mathrm{Si}$ sample. We thank $\mathrm{M}$. Birke, Ch. Niedermayer, and M. Pleines for their help in the initial phase of the experiment. The technical support by H.P. Weber is gratefully acknowledged.

*Electronic address: thomas.prokscha@psi.ch

${ }^{\dagger}$ Present address: Zentralabteilung Technologie, FZ Jülich GmbH, D-52425 Jülich, Germany.

[1] B. D. Patterson, Rev. Mod. Phys. 60, 69 (1988).

[2] K. H. Chow, B. Hitti, and R. F. Kiefl, in Semiconductors and Semimetals, edited by M. Stavola (Academic, San Diego, 1998), Vol. 51A.

[3] S. F. J. Cox, J. Phys. Condens. Matter 15, R1727 (2003).

[4] R. N. Pereira et al., Phys. Rev. B 72, 115212 (2005).

[5] G. Lüpke et al., Phys. Rev. Lett. 88, 135501 (2002).

[6] J. M. Gil et al., Phys. Rev. B 64, 075205 (2001).

[7] S. F. J. Cox et al., Phys. Rev. Lett. 86, 2601 (2001).

[8] E. A. Davis, S. F. J. Cox, R. L. Lichti, and C. G. V. de Walle, Appl. Phys. Lett. 82, 592 (2003).

[9] C. G. Van de Walle and J. Neugebauer, Nature (London) 423, 626 (2003).

[10] For the investigated insulators the mean energy $E_{e h}$ for electron-hole pair creation ranges from $18 \mathrm{eV}\left(\mathrm{SiO}_{2}\right)$ to $40 \mathrm{eV}$ for $\mathrm{Ne}$ (Ref. [19], Vol. II, p. 1136). For Si, $E_{e h} \simeq$ 3.7 eV, see F. E. Emery and T. A. Rabson, Phys. Rev. 140, A2089 (1965).

[11] E. Krasnoperov et al., Phys. Rev. Lett. 69, 1560 (1992).

[12] V. Storchak et al., Phys. Rev. Lett. 78, 2835 (1997).

[13] D. G. Eshchenko, V. Storchak, and G. D. Morris, Phys. Lett. A 264, 226 (1999).

[14] D. G. Eshchenko et al., Phys. Rev. B 66, 035105 (2002).

[15] D. G. Eshchenko, V. G. Storchak, S. P. Cottrell, and S. F. J. Cox, Phys. Rev. B 68, 073201 (2003).

[16] E. Morenzoni et al., Phys. Rev. Lett. 72, 2793 (1994).

[17] E. Morenzoni et al., Physica (Amsterdam) 326B, 196 (2003).

[18] J. D. Brewer et al., Physica (Amsterdam) 289-290B, 428 (2000).

[19] Rare Gas solids, edited by M. L. Klein and J. A. Venables (Academic, New York, 1975), Vol. I and II.

[20] T. Prokscha et al., Physica (Amsterdam) 326B, 51 (2003).

[21] R. A. Baragiola, M. Shi, R. A. Vidal, and C. A. Dukes, Phys. Rev. B 58, 13212 (1998).

[22] E. Morenzoni et al., J. Phys. Condens. Matter 16, S4583 (2004).

[23] P. W. Peakcock and J. Robertson, Appl. Phys. Lett. 83, 2025 (2003). 